\title{
INTERNACIONALIZACIÓN DEL CURRÍCULO UNIVERSITARIO VIRTUAL EN EL CONTEXTO DE LA GLOBALIZACIÓN
}

\author{
Internationalization of the virtual university curriculum in the context \\ of globalization
}

\section{Michel Valdés Montecinos*}

\section{RESUMEN}

Año tras año aumenta a nivel mundial la cantidad de estudiantes de educación superior, y de manera particular en la modalidad virtual, ante esta realidad se conjugan una serie de fenómenos que han impulsado a las instituciones universitarias a reinventarse. El objetivo de este trabajo es identificar la influencia que ejercen la globalización y la internacionalización en el currículo universitario de la educación superior, haciendo particular énfasis en la educación virtual latinoamericana. La metodología empleada es la revisión de bibliografía especializada en la temática y documentos oficiales de organismos involucrados. Los resultados revelan que: 1) Organismos multilaterales han desplegado esfuerzos por establecer acuerdos de asociación y cooperación académica conducentes a dos vías; por un lado, favorecer la movilidad de estudiantes y profesores, así como la realización de proyectos conjuntos; por el otro, impulsar los procesos de control de calidad e internacionalización del currículo. 2) En lo que respecta a la educación virtual en la región se ha fijado la mirada en la necesidad de asegurar y demostrar la calidad de sus programas, siendo el Instituto Latinoamericano y del Caribe de Calidad en Educación Superior a Distancia (CALED) una de las principales referencias en cuanto a directrices e instrumentos para la evaluación y asesoría a las universidades sobre los procesos de evaluación de la calidad y acreditación. Se concluye que la internacionalización del currículo en la educación universitaria virtual en América Latina tiene ante sí el desafío de dar el paso hacia la internacionalización comprehensiva, es decir, aquella que impacta de forma integral el plan de estudio a partir de una estructura conceptual y cultural que incluya la interdisciplinariedad y la multiculturalidad.

Palabras clave: Aprendizaje en línea; Enseñanza Superior; Universidad Internacional.

\footnotetext{
* Universidad Arturo Prat. Magister de Educación Superior, mención Docencia Universitaria, San Pablo 1796. Santiago Región Metropolitana, Chile. Email: michelvaldes@unap.cl
} 


\section{ABSTRACT}

Year after year the number of students in higher education increases worldwide, and particularly in the virtual mode. In the face of this reality, a series of phenomena combine that have driven university institutions to reinvent themselves. The objective of this work is to analyze the influence of globalization and internationalization on the curriculum of university education, with particular emphasis on Latin American virtual education. The methodology used is the review of both literature specialized in the subject and official documents of the agencies involved. The results reveal that: 1) multilateral agencies have been made efforts to establish two-way academic partnership and cooperation agreements, on the one hand, to promote the mobility of students and teachers, as well as the realization of joint projects; on the other hand, to promote the processes of quality control and internationalization of the curriculum. 2) Regarding virtual education in the region, the need to ensure and demonstrate the quality of its programs has been set, with the Latin American and Caribbean Institute of Quality in Distance Higher Education (CALED) being one of the main references regarding guidelines and instruments for evaluation and advice to universities on quality assessment and accreditation processes. It is concluded that the internationalization of the curriculum in virtual university education in Latin America faces the challenge of taking the step towards comprehensive internationalization, that is, the one that comprehensively impacts the curriculum from a conceptual and cultural structure including interdisciplinary studies and multiculturalism.

Keywords: Online learning; Higher Education; International University

\section{Introducción}

La virtualización de la educación a distancia viene aparejada al inicio del nuevo milenio y a la utilización intensiva de nuevas tecnologías de la información y la comunicación en el contexto de un proceso de expansión de la educación pública acontecida en toda la región y especialmente, en México, Brasil, Venezuela, Argentina y Perú. Este proceso ha impulsado la transformación de la tradicional educación a distancia semipresencial basada en recursos de aprendizaje en papel a la incorporación de plataformas de aprendizaje con el consecuente aumento de proveedores, y la necesidad de una diferenciación curricular y pedagógicas aplicables a nuevas tipologías de instituciones. 
Internacionalización del currículo universitario virtual en el contexto de la globalización

Según Rama (2011), para el año 2000 la región tenía 168.000 estudiantes en educación a distancia...bajo modelos pedagógicos a distancia o semipresenciales, que representaban el 1,3\% de la matrícula regional. A partir de entonces se produjo un salto, con un crecimiento interanual del $24 \%$ entre el 2000 y el 2010 alcanzando una matrícula de 1,5 millones de estudiantes, que representaron el 7\% de la cobertura regional, con un predominio de modelos semipresenciales, una predominancia privada y una tasa superior de crecimiento de la educación semivirtual respecto a la tradicional de educación a distancia.

Todo ello conlleva a la universidad la necesidad de plantearse cambios, no sólo de su formato y estructura clásicos sino de su propio enfoque de la educación en el marco de movimientos a escala internacional que presionan para que las instituciones den respuesta a los condicionamientos. En este sentido, el objetivo de este trabajo es analizar la influencia que ejercen la globalización y la internacionalización en el currículo universitario de la educación superior, haciendo particular énfasis en la educación virtual latinoamericana. La metodología empleada es la revisión de bibliografía especializada en la temática y documentos oficiales de organismos involucrados.

\section{Internacionalización y Globalización}

La expansión de redes internet alrededor de todo el mundo forma parte de las transformaciones estéticas, culturales y económicas que constituyen el proceso conocido como globalización, caracterizado por un agresivo empoderamiento de actores transnacionales (grandes empresas), un debilitamiento de los Estados en su ejercicio soberano y una emergencia de nuevos actores sociales (movimientos de mujeres, indígenas, jóvenes, organizaciones de derechos humanos). Estos fenómenos son paralelos a una reconfiguración de lo que hace poco conocíamos como espacio público (esfera que se circunscribía al espacio nacional concebido como campo de interacción entre los ciudadanos y el Estado). Internet es una herramienta estratégica para la construcción de 
este nuevo espacio público en el que compiten actores de diferentes niveles (globales, regionales, nacionales o locales).

Este fenómeno suele estar asociado con otro término denominado internacionalización, que si bien aparecen de manera obligada en análisis de contexto, tienen desde el punto de vista conceptual diferentes acepciones. Según Knight y Wit (1997), la globalización se refiere al flujo de conocimientos, tecnología, personas, valores, ideas que trascienden las fronteras, fenómeno que afecta a cada país de manera diferente, en relación con su historia, tradiciones, cultura y prioridades. Por su parte Altbach, et al. (2009), afirman que la globalización es una realidad clave en el siglo $\mathrm{XXI}$, que ha influido de forma profunda en la enseñanza superior. Estos autores definen la globalización como la realidad formada por una economía mundial cada vez más integrada, las nuevas tecnologías de la información y la comunicación, la aparición de una red de conocimiento internacional, el papel de la lengua inglesa y otras fuerzas más allá del control de las instituciones académicas.

Se trata entonces de un fenómeno que ha impactado todas las esferas, que trasciende la visión meramente económica, involucra todas las dimensiones sociales, superando las barreras geográficas y culturales. Teichler (2004), afirma que la globalización refiere a la totalidad de cambios sustanciales en el contexto y la vida interna de la enseñanza superior, relacionados con las crecientes interrelaciones entre diferentes partes del mundo con las que las fronteras nacionales se difuminan o incluso parecen desaparecer.

La internacionalización en el contexto de la educación superior comenzó a introducirse sistemáticamente a principios de los ochenta, como respuesta a los retos planteados por la globalización. En este sentido, la internacionalización es entendida como la respuesta social, cultural y educativa de los países al impacto de la globalización, y a través de ella, el mundo académico pretende enfrentar de forma 
Internacionalización del currículo universitario virtual en el contexto de la globalización

proactiva la mundialización, salvaguardando las particularidades locales (Altbach y Knight, 2006); (Knight, 2008); (Altbach et al, 2009); (León y Madera, 2016).

La internacionalización de las universidades no es un fenómeno nuevo, aunque en los últimos años se ha convertido en objeto de debates y manifiesta una nueva dinámica institucional (Araya y Oregioni, 2015). Teniendo en cuenta lo anterior se puede afirmar que la internacionalización es un tema de interés permanente para las intituciones de educación superior (IES) y sobre todo para las universidades. Según Hoyos-Villa (2015) en los últimos tres siglos (XIX, XX y XXI), por el gran interés en la internacionalización de parte de las universidades, se ha generado una evolución importante en este aspecto, hasta constituirse hoy en día en un proceso absolutamente necesario para la actuación de las IES (Insituciones de educación superior) dentro del contexto de la globalización.

La internacionalización de la educación superior se trata entonces de un proceso que ha trascendido desde la visión de movilidad, para dar paso a otras aristas y la profundización de su significado; Haug (2010) señala que se internacionalizan los estudios, los campus, las instituciones de educación superior, aparejado con la búsqueda de una mayor calidad y relevancia en el contexto de una competitividad internacional más fuerte.

En este orden de ideas, la tendencia actual de los cambios que se vienen generando en materia de internacionalización de la educación superior sugiere transformaciones en distintos órdenes tanto en concepción del proceso como en su estructura. El llamado es a generar la denominada internacionalización comprehensiva, es decir, estrategias que impacten los tres niveles del proceso educativo: el macro (diseño de políticas y toma de decisiones); el mediano (estructuras y políticas curriculares) y el micro (proceso de enseñanza-aprendizaje). Solo siendo comprehensivas y transversales, las estrategias de internacionalización pueden contribuir al mejoramiento de la calidad y pertinencia educativa, y así apoyar los cambios que habiliten al sistema educativo a la nueva realidad global (Hudzik 2011; 
Gacel-Avila, 2012). Para efectos de esta investigación se pondra la mirada en el nivel meso, pero con plena conciencia de que se trata de un proceso que no se encuentra aislado de sus otros componentes.

\section{Internacionalización del currículo en educación superior}

La mejora continua del proceso educativo tal como se viene aludiendo ha tocado todas las esferas, siendo la internacionacinalización del curiculo un tema de particular interés. Si bien es importante proyectar visibilidad en las instituciones, esto pasa por revisar un aspecto medular del proceso de enseñanza-aprendizaje, cual es el currículo.

La internacionalización del currículo es definida como la integración de la dimensión internacional y multicultural en los contenidos y formas de los programas de curso, con la finalidad de formar egresados para actuar profesional y socialmente en un contexto internacional y multicultural. Este concepto también es considerado como una analogía del concepto "global", que se refiere al enriquecimiento del conocimiento de lo local desde una perspectiva global. Las medidas más comunes adoptadas por las IES, en este rubro son: Movilidad estudiantil, becas de estudio en el extranjero y participación de docentes e investigadores en redes internacionales de generación del conocimiento. (Gacel-Avila, 2014; Gacel-Avila, 2018).

Una definición que ofrece una perspectiva enfocada al aprendizaje del estudiante, como principal protagonista del proceso es la que ofrece Leask (2015), en tanto un curriculum internacionalizado compromete a los estudiantes con la investigación internacionalmente informada y la diversidad cultural y lingüística, además desarrolla deliberadamente sus perspectivas internacionales e interculturales como ciudadanos y profesionales globales. Con ello no se soslaya el proceso que se debe dar en otras esferas, sino que se hace énfasis en lo que se considera el fin y no el medio. 
Internacionalización del currículo universitario virtual en el contexto de la globalización

En este orden de cosas los modelos y estrategias para la internacionalización de las IES, pretenden perfilarse como opciones de "calidad" a las distintas formas de relaciones ínter-universitarias. Por lo tanto, el planteamiento es asegurar la calidad de los servicios y de las asociaciones, utilizando métodos de monitoreo, evaluación y acreditación. Hay un gran y renovado interés por someter a evaluación internacional programas e instituciones. Por lo anterior, el rol de las universidades en los procesos de internacionalización es relevante a la hora de establecer un parámetro común con respecto a la calidad en los procesos de aprendizajes en programas internacionales. En este sentido, se necesita evidenciar si las IES han estado a la altura del dinamismo de los cambios culturales y tecnológico de las personas, las industrias y de las sociedades. Altbach et al, (2009), en la Conferencia Mundial de Educación Superior señalaron que: Si bien, en el pasado, las universidades siempre habían estado influenciadas por las recomendaciones de los organismos internacionales; a principios de la década del siglo actual, de manera acelerada, las universidades se han adaptado a los retos que actualmente presentan la globalización económica y la sociedad de la información.

Las IES tienen un rol importante en ello, ante está responsabilidad los gobiernos y sus mesas de trabajo en conjunto con las instituciones multilaterales lo advirtieron hace ya más de dos décadas. Esto se vio expresado a través de los procesos y tratados europeos, donde aproximadamente veintinueve países europeos llegaron a acuerdos de meso currículos donde una de las variables a considerar es la internacionalización del currículo; la cual implica la implementación de estándares internacionales de calidad en la educación superior, para facilitar el reconocimiento de estudios que propendan a una articulación y homologación que finalmente pueda ejercer su profesión con excelencia en el ámbito local, nacional e internacional.

El ejemplo emblemático de estos acuerdos es el denominado proceso de Bolonia (1999), donde dos de sus temas centrales fueron las adaptaciones curriculares y adaptaciones tecnológicas en la educación; para posteriormente ser reforzado con la 
creación del Espacio Europeo de Educación Superior (EEES) y otros acuerdos en el mismo tenor como son:

- Comunicado de Praga (19 de mayo de 2001)

- Declaración de Salamanca (2001)

- Seminario de Helsinki (2001)

- Comunicado de Berlín (19 de septiembre de 2003)

- Comunicado de Bergen (19 y 20 de mayo de 2005)

- Comunicado de Londres (18 de mayo de 2007)

- Parlamento y Consejo Europeo (6 de mayo 2008)

- Declaración Leuven (2009)

- Declaración Budapest-Vienna (2010)

- Comisión Europea (2010)

- Declaración de Barcelona (2002)

- Declaración de Ereván (2015)

- Comisión Europea, EACEA y Eurydice, (2015)

El proceso de Bolonia ha constituido, ante todo, una oportunidad para la renovación curricular de los programas formativos que imparten las universidades ha inspirado todo un movimiento de reforma universitaria, particularmente en la innovación curricular; estos acuerdos son parte de la iniciativa emprendidas por un grupo de universidades europeas en verano del año 2000, grupo que aceptó colectivamente el reto formulado en Bolonia y elaboró un proyecto piloto denominado Tuning (Sintonizar las estructuras educativas de Europa). Los integrantes del proyecto pidieron a la Asociación Europea de Universidades (EUA) que les ayudara a ampliar el grupo de participantes, y solicitaron a la Comisión Europea una ayuda financiera en el marco del programa Sócrates ${ }^{1}$.

\footnotetext{
${ }^{1}$ El programa Sócrates fue una iniciativa educacional de la Comisión Europea; 31 países forman parte de él. La iniciativa del programa Sócrates lleva funcionando desde 1994 hasta el 31 Diciembre de 1999 cuando fue remplazado por el programa
} 
Internacionalización del currículo universitario virtual en el contexto de la globalización

El proyecto Tuning abordó varias de las líneas de acción señaladas en Bolonia y, en particular, la adopción de un sistema de titulaciones fácilmente reconocibles y comparables, la adopción de un sistema basado en dos ciclos y el establecimiento de un sistema de créditos. El proyecto Tuning contribuyó también a la realización de los demás objetivos fijados en Bolonia. Más concretamente, el proyecto se propuso determinar puntos de referencia para las competencias genéricas y las específicas de cada disciplina en una serie de ámbitos temáticos: estudios empresariales, ciencias de la educación, geología, historia, matemáticas, física y química.

Las competencias se definieron como puntos de referencia para la elaboración y evaluación de los planes de estudio, y no pretendieron ser moldes rígidos, permitiendo así dotar a los planes de estudio de flexibilidad y autonomía, pero, al mismo tiempo, introduciendo un lenguaje común para describir los objetivos de los mismos. (Espacio Europeo en Educación Superior, 2019).

Otro de los acuerdos que son de notable significación los (European Credit Transfer and Accumulation System (ECTS), como una serie de acuerdos intra-europeos de estandarización de los países europeos con el proceso de Bolonia. Los ECTS funcionaban como un sistema para garantizar el reconocimiento de créditos entre universidades europeas usando un criterio unificado de cálculo y proporcionando una prueba del rendimiento académico (Teichler, 2004), (Malet, 2017).

Ahora bien, en América Latina también se ha hecho sentir la influencia del proceso de Bolonia; en palabras de Cepeda y Gascón (2009) la Unión Europea ha venido elaborando estrategias para avanzar en la constitución de un espacio común de educación superior con Latinoamérica. Entre ellos destacan el proyecto 6x4 y AlfaTuning América Latina.

Sócrates II en 24 de enero de 2000, el cual siguió activo hasta el 2006. Éste, a su vez, fue reemplazado por el Lifelong Learning Programme 2007-2013. Sus objetivos: Fortalecer la dimensión europea de la educación a todos los niveles; Mejorar el conocimiento de las lenguas europeas; Promover la cooperación y la movilidad a través de la educación; Alentar la innovación en la educación; Promover la igualdad de oportunidades en todos los sectores de la educación. Desde el año 2014 el programa continúa denominado Erasmus+ como parte de la Estrategia Europea 2020. 
El primero responde al propósito principal de la Declaración de la Conferencia Ministerial de los países de la Unión Europea, de América Latina y el Caribe sobre la Enseñanza Superior (París, noviembre de 2000), que es la construcción de un espacio común de educación superior UEALC $6 \times 4^{2}$ que se refiere a seis profesiones en cuatro ejes de análisis. Los aspectos clave son el desarrollo de un acercamiento a la evaluación y reconocimiento de los resultados del aprendizaje, expresados en términos de competencias, y el fortalecimiento de la pertinencia y de los vínculos de la educación superior y la investigación con la sociedad en el marco de la colaboración entre países y regiones. Se puso especial atención en el desarrollo de mecanismos para facilitar el reconocimiento de las calificaciones y competencias de las personas, tanto para continuar con sus estudios y su vida laboral como para incrementar la movilidad académica (Proyecto 6x4 UELAC, 2008).

En el segundo caso, Tuning Latinoamérica surgió en el año 2004, este proyecto se autodefine como un espacio de reflexión de actores comprometidos con la educación superior que la búsqueda de consensos, contribuye avanzar en el desarrollo de titulaciones fácilmente comparables y comprensibles de forma articulada en América Latina (Tuning Project, 2007). Adicionalmente sostiene Navarro Leal (2014) el Proyecto Alfa Tuning América Latina buscó comprender y ser la respuesta a los procesos actuales de globalización, impulsando los procesos de control de la calidad e internacionalización del currículo y planes de estudios (Ver gráfico 1);

\footnotetext{
${ }^{2}$ Administración; Ingeniería Electrónica o similar; Medicina; Química; Historia; Matemáticas y los cuatro ejes de análisis: Competencias profesionales; Créditos académicos; Evaluación y acreditación; Formación para la innovación y la investigación.
} 
Internacionalización del currículo universitario virtual en el contexto de la globalización

\section{Gráfico 1. Alcances de la Internacionalización del Currículo}

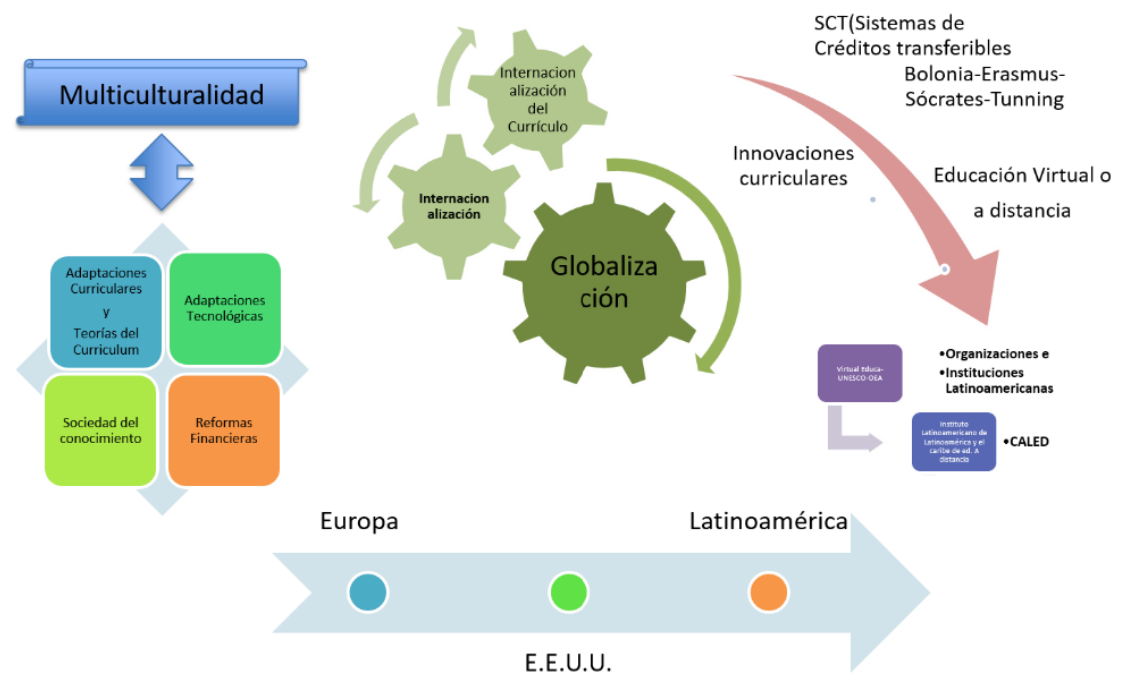

Fuente: Valdés et al. (2015)

Por otra parte se han desplegado acuerdos de asociación y cooperación, desde organismos como ALADI (Asociación Latinoamericana de Integración) y UNASUR (La Unión de Naciones Suramericanas), lo que ha generado la necesidad de ampliar la colaboración científica y académica. Una de las propuestas de estas alianzas es internacionalizar el currículo para así responder a necesidades, no sólo locales, sino que también subcontinentales, como una herramienta necesaria en busca de estos nuevos desafíos (UNESCO, 2015).

En los últimos años los países latinoamericanos han establecido objetivos nacionales para las políticas sobre educación terciaria, políticas a nivel de cada nación y políticas de bloque de cooperación para el establecimiento de normas comunes como el espacio educacional MERCOSUR ${ }^{3}$. Se reconoce al mismo tiempo, un número de

\footnotetext{
${ }^{3}$ El Sector Educativo del MERCOSUR es un espacio de coordinación de las políticas educativas que reúne los países miembros y asociados del MERCOSUR, desde diciembre de 1991, cuando el Consejo del Mercado Común (CMC creó la Reunión de Ministros de Educación del MERCOSUR (RME). Con el tiempo, la Reunión de Ministros creó otras instancias para apoyar el Sector, en 2001 se aprobó la "estructura organizativa del Sector Educativo del MERCOSUR", que crea el
} 
problemas comunes existentes en el sistema de educación superior, acceso a la educación de ciudadanos de bajos quintiles de ingresos, con el objeto de generar movilidad social; tener una educación de calidad; contribuir al desarrollo nacional, en las ciencias, en las artes y en las tecnologías; todas estas, propuestas para innovar y mejorar la productividad del país, con el objeto de aumentar las mejores condiciones de vida de la sociedad y disminuir sistemáticamente los índices de pobreza.

La factibilidad de construir un espacio común de educación superior en América Latina, a través de un proceso como el de Bolonia o alguna de sus variantes, es un tema de debate en la región. A pesar de que se reconocen sus bondades en términos de modernización, innovación e internacionalización, sobresale la tesis de que en el caso de América Latina no es factible. Los argumentos en que se sustenta esta tesis son, por una parte, el riesgo de homogenizar los sistemas de educación superior en todas las regiones, lo que pondría en peligro la diversidad y la diferenciación regional, y, por otra parte, las asimetrías de tipo intrarregional que existen entre los sistemas de la región, así como las de tipo interregional, que se refieren a la falta de un macroproyecto de integración económica, política y social, aunado esto a las diferencias existentes entre las características de la educación superior de América Latina y de Europa (GacelAvila, 2011).

Agrega Rodríguez (2014) que si bien las universidades latinoamericanas no cuentan aún con las estructuras organizativas adecuadas que les permitan organizar e integrar las actividades internacionales para mejorar sus funciones sustantivas, y las universidades con sus diversas actividades académicas, proyectos y programas no han terminado de insertar las políticas y prácticas de la implementación de currículos

Comité de Coordinación Regional, las Comisiones Regionales Coordinadores de Área (Básica, Tecnológica y Superior) y el Comité Gestor del Sistema de Información y Comunicación. En 2005 se creó el Comité Asesor del Fondo Educativo del MERCOSUR. En 2006, se creó la Red de Agencias Nacionales de Acreditación, y en 2011 se creó la Comisión Regional Coordinadora de Formación Docente. Además de estos, hay otras instancias, temporales y permanentes, que gestionan acciones específicas. A través de la negociación de políticas públicas y la elaboración y ejecución de programas y proyectos conjuntos, el Sector Educativo del MERCOSUR busca la integración y el desarrollo de la educación en toda la región del MERCOSUR y países asociados. 
Internacionalización del currículo universitario virtual en el contexto de la globalización

regulares, el proceso de internacionalización tiene un gran potencial en este sentido; ante lo cual, las diversas agrupaciones universitarias y los organismos internacionales se están esforzando en este camino.

A raíz de estos tratados y comunicados se puede deducir la importancia del desarrollo de competencias en las personas, en especial para prepararlos como profesionales integrales, además se profundizó el diseño curricular. "Si los estudiantes no desarrollan las competencias necesarias para funcionar de manera eficiente en un entorno global, es remoto que tengan éxito en el mercado laboral y en la sociedad del Siglo XXI" (Shoenberg, 1998). Además contemplando las competencias a desarrollar y la internacionalización, las IES se vieron en la obligación a ampliar su misión, desde una institución que produce y socializa el conocimiento científico, a la de preparar ciudadanos del futuro para un mundo interconectado e interdependiente.

\section{Internacionalización y Educación virtual}

Se está inaugurando la etapa de la educación continua, que inicia con el nacimiento y se prolonga por toda la vida. Es decir, el horizonte de educación será igual a la expectativa de vida en cada sociedad. Así se denota hace ya más de una década en informes de instituciones multilaterales como lo son UNESCO a través del informe de la Conferencia Regional de Educación Superior, de Virtual Educa y sus Congresos anuales e informes del Banco Mundial a través de sus informes anuales; además se confirma en Congresos y Seminarios regionales, como por ejemplo, el Congreso Internacional de Innovación Educativa (CIIE), organizado por el Tecnológico de Monterrey, cuyo temas más recurrentes y de interés son la internacionalización, la educación continua o educacion para toda la vida, y la educación a distancia, en especial la modalidad virtual de aprendizaje.

Las modalidades virtuales surgen como respuesta a la necesidad de especialización de muchas áreas de trabajo, la masividad que proporciona esta herramienta acerca al conocimiento especializado y a la formación de capital humano avanzado, esto ha generado la apertura de varios programas favoreciendo 
considerablemente la cobertura y accesibilidad de la educación post gradual, independientemente del lugar geográfico donde se encuentre el estudiante.

Claudio Rama (2013), en este gráfico muestra como la masificación es un tramado en busca de la internacionalización como respuesta a la globalización y a la vez constituye una tendencia o macrotendencia en la educación superior en América Latina, siendo así una dinámica constante con cambios en sus diferentes ámbitos.

Imagen $N^{\circ} 2$ Macrotendencias de la educación superior en América Latina

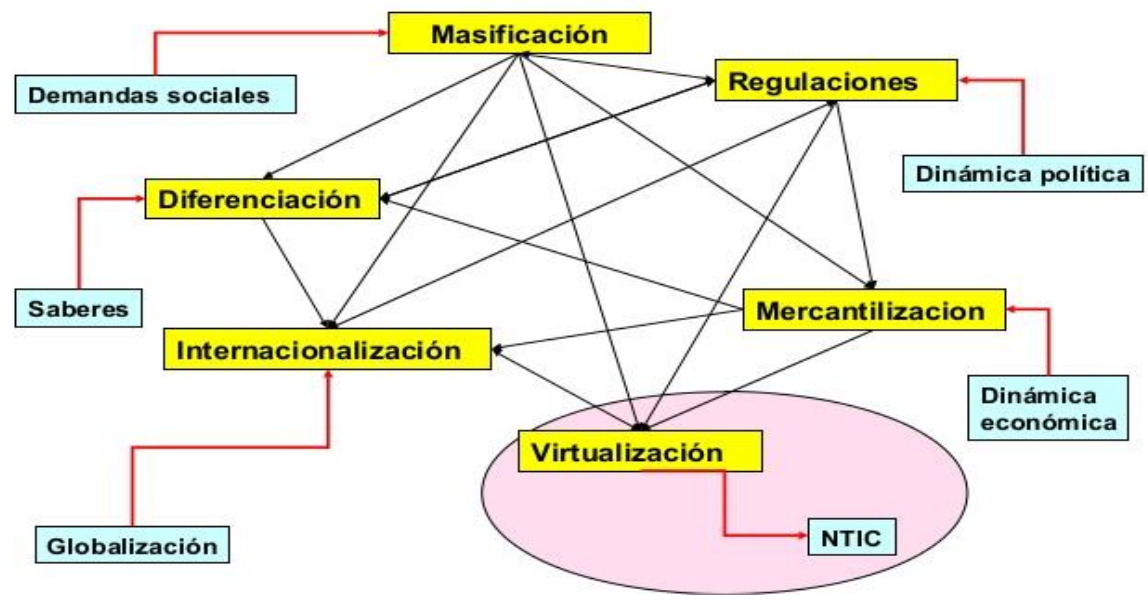

Fuente: Rama (2013)

Este autor agrega que la virtualización constituye un factor que resalta entre el conjunto de determinantes en la internacionalización de la educación, al facilitar que el fraccionamiento de los procesos educativos a distancia adquiera dimensiones internacionales transfiriendo y terciarizando partes de los insumos y procesos educativos Rama (2012). La virtualización puede ser entendida como la representación de procesos y objetos asociados a actividades de enseñanza y aprendizaje, investigación, extensión y gestión, así como objetos cuya manipulación permite al 
Internacionalización del currículo universitario virtual en el contexto de la globalización

usuario, realizar diversas operaciones a través de internet, tales como, aprender mediante la interacción con cursos electrónicos, inscribirse en un curso, consultar documentos en una biblioteca electrónica, comunicarse con estudiantes, profesores y otros (Silvio, 2000).

Agrega Chan (2016), que la virtualización educativa no está limitada a la representación de objetos, escenarios y experiencias del mundo real para colocarlos en entornos digitales, eso es sólo una dimensión. También se opera en el modo inverso, en el que la mediación tecnológica está interviniendo los objetos y espacios físicos convirtiéndolos en dispositivos de información, conocimiento y comunicación.

Para Rama (2012), la confluencia de diversos factores está contribuyendo a una dinámica articulada de la internacionalización con la educación a distancia. Ella se realiza tanto asociada a procesos de virtualización como en el marco de las modalidades tradicionales semipresenciales de la educación a distancia. Así, al tiempo que aumenta la presencia de instituciones de educación a distancia y que aumenta la cobertura local de estudiantes bajo estas modalidades, se produce la internacionalización de algunas de estas instituciones y de los procesos educativos asociados, tanto por presiones competitivas como por aprovechar ventanas de oportunidades que se generan en costos y calidad.

Si bien la educación a distancia ha estado aparejada al fenómeno de la internacionalización, o se evidencian experiencias que desarrollen la internacionalización del currículo en la educación virtual, los esfuerzos se han centrado en el sistema de aseguramiento de la calidad.

\section{Aseguramiento de la calidad para la educación virtual}

La educación bajo la modalidad virtual, como ya se dijo anteriormente, ha crecido de forma exponencial, y con ello, la proliferación de programas que no necesariamente cumplen criterios de calidad. En opinión de Duart y Sangrá (2000), en el ámbito 
universitario, la emergencia de la formación virtual, o del e-learning, lejos de aparecer como una oportunidad de desarrollo y de puesta al día respecto de las necesidades de la sociedad, está siendo percibida como una amenaza por algunas instituciones universitarias. Este temor ha generado como mecanismo de defensa un discurso dubitativo, cuando no peyorativo, hacia este tipo de formación universitaria, cuestionando su calidad. En ese tenor señala Moreno (2007), que aunque parezca una obviedad, la calidad no depende de la modalidad, pues ésta puede ser tan buena o mala como la presencial. La calidad depende más de sus procesos educativos y éstos fundamentalmente de las personas que participan en ellos: estudiantes, profesores, tutores, técnicos, directivos, entre otros.

Aunado a ello, está presente la necesidad de que las credenciales o títulos otorgados sean reconocidos internacionalmente, tal como se había aludido en el apartado anterior. Por ambas razones, varios países de América Latina han unido esfuerzos a fin de establecer procesos de evaluación nacional de cualificaciones. Señalan Bañuelos y Montero (2017), que al realizar la evaluación de un programa educativo virtual, se puede determinar la forma en que se están desarrollando sus procesos, en busca de una mejora de la calidad educativa o con fines de acreditación. Han nacido también organismos cuyo principal objetivo es asegurar y privilegiar la calidad en la educación internacional, especialmente en lo que es la dimensión internacional de la educación superior.

Actualmente, se observa una creciente tendencia a aceptar que los criterios de calidad estén siendo cada vez más definidos en términos de normas y estándares internacionales, lo que pueden observarse en el trabajo de agrupaciones profesionales, agencias de acreditación, organizaciones no gubernamentales, entre otros. Una de las instituciones que se encarga de asegurar la calidad de la educación es el Instituto Latinoamericano y del Caribe de Calidad en Educación Superior a Distancia (CALED), el cual brinda asesoría en los procesos de autoevaluación a toda casa de estudio que lo requiera. Esta entidad tiene su origen en el Congreso sobre Calidad y Acreditación Internacional en Educación Superior a Distancia, celebrado en octubre del 2005 en la 
Internacionalización del currículo universitario virtual en el contexto de la globalización

Universidad Técnica Particular de Loja (Ecuador), el cual despertó el interés de un gran número de instituciones que resuelven su creación.

La citada organización ha generado un tarjeta de valoración de la calidad (SCCQAP), diseñada con el propósito de medir y cuantificar elementos de calidad dentro de los programas de educación en línea en la educación superior; la cual constituye una herramienta práctica al momento de realizar un proceso de evaluación, ya que permite a través de un conjunto de 9 categorías y 91 indicadores determinar la calidad del programa objeto de estudio. Con este fin el Online Learning Consortium (OLC) y el Instituto Latinoamericano y del Caribe de Calidad en Educación Superior a Distancia (CALED), realizaron un proceso combinado y colaborativo que enriqueció y complementó cada una de sus propias metodologías de evaluación (CALED, 2015).

Cuadro 1. Estructura del modelo: tarjeta de puntuación para la evaluación de programas en línea

\begin{tabular}{|c|c|c|}
\hline & Categorías & Indicadores \\
\hline 1 & Apoyo Institucional & 8 \\
\hline 2 & Apoyo Tecnológico & 8 \\
\hline 3 & $\begin{array}{c}\text { Desarrollo y diseño instruccional de los } \\
\text { cursos en línea }\end{array}$ & 20 \\
\hline 4 & Estructura de los cursos en línea & 9 \\
\hline 5 & Enseñanza y aprendizaje & 6 \\
\hline 6 & Participación social y estudiantil & 1 \\
\hline 7 & Apoyo a los docentes & 6 \\
\hline 8 & Apoyo a los alumnos & 20 \\
\hline 9 & Evaluación y valoración & 13 \\
\hline & Total & $\mathbf{9 1}$ \\
\hline
\end{tabular}

Fuente: CALED (2015).

Vale decir que este modelo fue concebido para evaluar programas de pregrado en línea, pero ante la inexistencia de un modelo aplicable al caso de postgrado, se utiliza éste de forma genérica. Por lo tanto, se hace necesario generar herramientas que se enfoquen en verificar si existen criterios aplicables a las particularidades de los estudios de postgrado. 
En suma, puede decirse que los nuevos tiempos exigen las universidades que ofrecen formación basada en la web tal como señalan Duart y Sangrá (2000), a cuestionarse su modelo organizativo, al tiempo que también se hace necesario el paso de la docencia convencional a la docencia mediante la web, y esto aporta al profesorado la posibilidad de plantearse de nuevo la labor docente.

La situación actual exige a las instituciones una educación en red y digital tal como lo califica Rama (2019), con aprendizaje flexible y un carácter global. La universidad y la educación ya no son completamente nacionales, ni lo son los estudiantes, profesores, currículos, recursos de aprendizaje ni mucho menos, los conocimientos, las pertinencias ni los ejercicios laborales. Efectivamente se inaugura una nueva lógica, la de la movilidad y migración estudiantil, docente y profesional para acceder y transferir conocimiento en el contexto actual.

\section{Conclusiones}

La internacionalización se presenta como la respuesta social, cultural y educativa de los países al impacto de la globalización, y a través de ella, el mundo académico pretende enfrentar de forma proactiva la mundialización salvaguardando las particularidades locales. Por ende, (López, 2018), hace el llamado a que los valores tradicionales de la Universidad sigan siendo válidos (la autonomía, la libertad de cátedra, la investigación, el trabajo de los estudiantes, la evaluación), debido a que son valores amenazados en el contexto de la globalización, al cuestionarse el ideal de lo público y del bien común.

Desde la década de los noventa asistimos a un movimiento planetario con la intención de establecer acuerdos de asociación y cooperación académica conducentes a dos vías, por un lado, favorecer la movilidad de estudiantes y profesores, así como la realización de proyectos conjuntos; por el otro, impulsar los procesos de control de calidad e internacionalización del currículo. 
Internacionalización del currículo universitario virtual en el contexto de la globalización

En lo que respecta a la educación virtual en la región se ha fijado la mirada en la necesidad de asegurar y demostrar la calidad de sus programas, siendo el Instituto Latinoamericano y del Caribe de Calidad en Educación Superior a Distancia (CALED), una de las principales referencias en cuanto a directrices e instrumentos para la evaluación y asesoría a las universidades sobre los procesos de evaluación de la calidad y acreditación.

El énfasis de CALED en su modelo de evaluación se ha dirigido hacia el nivel de pregrado en línea, sin que se hayan desarrollado modelos aplicable al nivel de postgrado para dar respuesta a sus posibles especificidades. Los esfuerzos emprendidos hasta ahora en educación virtual evidencian avances en lo que a infraestructura se refiere, es decir en favorecer una interacción efectiva entre participantes, docentes y personal de apoyo, corresponde dar el salto hacia la profundización de currículo, en términos de lo que se conoce como internacionalización comprehensiva, es decir, aquella que impacta de forma integral el plan de estudio a partir de una estructura conceptual y cultural que incluya la interdisciplinariedad y la multiculturalidad; en definitiva, una institución fortalecida para los acceder y transferir conocimiento en el contexto actual.

\section{Referencias Bibliográficas}

Araya, José María y Oregioni, María Soledad (2015). Internacionalización de la Universidad en el marco de la Integración Regional. Universidad Nacional del Centro de la Provincia de Buenos Aires.Argentina

Altbach, Philip y Knight, Jane (2006). Visión panorámica de la internacionalización en la educación superior: motivaciones y realidades. Perfiles Educativos, Revista del Instituto de Investigaciones sobre Universidad y Educación. Universidad Nacional Autónoma de México. Vol. 28, N 12, México. (Pp. 13-39).

Altbach Philip; Reisberg, Liz y Rumbley, Laura. (2009) Trends in Global Higher Education: Tracking an Academic Revolution A Report Prepared for the UNESCO. UNESCO. France.

Bañuelos Márquez, Ana María y Montero Montiel Gabriela (2017) La evaluación de la educación a distancia. Propuesta de una guía para la autoevaluación. Hamut'ay. Revista semestral de divulgación científica División de 
Investigación y Extensión Científica Tecnológica (DIECT-DUED). Vol. 4(1), Perú (Pp. 31-44).

CALED (2015). Instituto Latinoamericano y del Caribe de Calidad en Educación Superior a Distancia. El Proceso de Garantía de Calidad para la Educación en Línea y a Distancia OLC /CALED. TARJETA DE PUNTUACIÓN (SCCQAP). EVALUACIÓN DE PROGRAMAS DE PREGRADO EN LÍNEA. Universidad Técnica Particular de Loja. Ecuador.

Cepeda Dovala, José Luis y Gascón Muro, Patricia (2009). El Proceso de Bolonia en América Latina: Caso México. Disponible en: http://www.institutgouvernance.org/es/analyse/fiche-analyse-431.html. Consulta: 25-01-19.

Chan, Ana María (2016). La virtualización de la educación superior en América Latina: entre tendencias y paradigmas. RED-Revista de Educación a Distancia. Extraído de: https://www.um.es/ead/red/48/chan.pdf consulta: 25-01-19.

Duart, Joseph y Sangrá Albert (2000). Formación universitaria por medio de la web: un modelo integrador para el aprendizaje superior. En: Aprender en la virtualidad Editorial Gedisa. España

Espacio Europeo en Educación Superior (2019). Estructuras educativas europeas. La respuesta de la universidad mediante el proyecto «Tuning». Disponible en: http://www.eees.es/es/eees-estructuras-educativas-europeas consulta: 19-0719.

Gacel-Avila, Jocelyne (2011). «Impacto del proceso de Bolonia en la educación superior de América Latina». En: «Globalización e internacionalización de la educación superior» [monográfico en línea]. Revista de Universidad y Sociedad del Conocimiento (RUSC). Vol. 8, n. ${ }^{\circ}$ 2, Suiza (Pp.123-134).

Gacel-Avila Jocelyne (2012). La educación terciaria en América Latina. El concepto de internacionalización comprehensiva En: la internacionalización de la educación superior a nivel mundial y regional. Principales tendencias y desafíos. Compiladores. Humberto Grimaldo Durán, Francisco López Segrera. Editorial Planeta. Colombia.

Gacel-Avila, Jocelyne (2014). Internacionalización del currículo. Seminario:Responsabilidad y Rehumanización, obligaciones territoriales. Observatorio Regional de Responsabilidad Social para América Latina y el Caribe (ORSALC). México.

Gacel-Avila, Jocelyne (2018). La internacionalización de la educación terciaria en América Latina y el Caribe. En: La educación superior, internacionalización e integración en América Latina y el Caribe. Balance regional y prospectiva UNESCO - IESALC y UNC. España.

Haug, Guy (2010). La internacionalización de la educación superior: más allá de la movilidad europea. La Cuestión Universitaria, Boletín electrónico de la 
Internacionalización del currículo universitario virtual en el contexto de la globalización

cátedra UNESCO de política y gestión universitaria. número 6. España. (Pp. 20-29).

Hoyos-Villa, Oscar (2015). Modelo de Internacionalización de las IES del Eje Cafetero Colombiano como aporte al Desarrollo Regional. Tesis Doctoral Universidad de Nebrija. España

Hudzik, John (2011). Comprehensive Internationalization. From Concept to Action. NAFSA: Association of International Educators. United States of America.

Knight, Jane (2008). Higher Education in Turmoil. The Changing World of Internationalization. Sense Publishers. United States of America.

Knight, Jane y De Wit, Hans. (1997). Internationalization of higher education in Asia Pacific countries. European Association for International Education. Australia.

Navarro Leal, Marco (2014). Internacionalizacion y educacion superior. Bloomington: Palibrio.Estados Unidos.

Leask Betty (2015). Internationalizing the Curriculum. Routledge. Inglaterra.

León Robaina, Rosario y Madera Soriano, Luz (2016). La internacionalización universitaria, un imperativo de la educación superior en el contexto latinoamericano actual. Encuentros, 14(2). Colombia (Pp. 43-59).

López Segrera (2018). Retos de la educación superior en un contexto de incertidumbre y crisis global. Avaliacão: Revista da Avaliação da Educação Superior (Campinas). vol.23 no.2. Brasil. (Pp. 551-566).

Malet Calvo, Daniel (2017). Globalización e internacionalización educativa. Una historia institucional del Programa ERASMUS, 1987-2014. Ler História, Número 71, Portugal. (Pp. 75-100).

Moreno Castañeda, Manuel (2007). La calidad de la educación a distancia en ambientes virtuales. Apertura. Revista de Innovación Educativa. Universidad de Guadalajara. Número 6. México. (Pp. 19-31).

Proyecto 6x4 UEALC (2008). Resumen ejecutivo 2004-2007. Disponible en: http://108.59.253.179/ uealc/site2008/pre/03.pdf consulta: 05-04-19.

Rama, Claudio. (2011). La educación superior en América Latina en el periodo 20002010: ocho ejes centrales en discusión, Revista Innovación Educativa, vol. 11, núm. 57. México. (Pp. 15-20).

Rama Claudio (2012). La internacionalización de la educación a distancia en América Latina. Cuestiones de sociología. $\mathrm{N}^{\circ} 8$. Argentina. (Pp. 1-14).

Rama, Claudio (2013). Las tendencias de la educación a distancia en un mundo global.

I Seminario Internacional: "E - Learning: Un proceso innovador" Universidad Inca Gracilazo de la Vega - Facultad de Ciencias Administrativas y Económicas.Lima, Perú.

Rama, Claudio (2019). Políticas, tendencias y tensiones de a educación a distancia y virtual en América Latina. Ediciones Universidad Católica de Salta. Argentina. 
Rodríguez Betanzos, Addy (2014). Internacionalización curricular en las universidades latinoamericanas. Revista argentina de educación superior, N $^{\circ} .8$. Argentina. (Pp. 149-168).

Shoenberg, Robert (1998). Next Steps for Languages Across the Curriculum: Prospects, Problems, \& Promise. American Council on Education. United States of America.

Silvio José (2000). La virtualización de la universidad: ¿Cómo transformar la educación superior con la tecnología? IESALC/UNESCO. Venezuela.

Teichler, Ulrich (2004). The Changing Debate on Internationalization of Higher Education. Higher Education, Vol.48, $\mathrm{N}^{\circ}$ 1. Holanda. (Pp. 5-26).

Tuning Project (2007). Reflexiones y perspectivas de la educación superior en América Latina. Informe final 2004-2007. Universidad de Deusto. España.

UNESCO. (2015). Science report: towards 2030. Disponible en: https://es.unesco.org/unesco_science_report. Consulta: 26-04-19.

Valdés, Michel; Chavez, Norma y Ossandon, Carlos. (2015). Formación de Capital Humano Avanzado "El desafío del hoy". Virtual Educa Seminario "La internacionalización de la educación superior en la sociedad del conocimiento. Coordinación de Universidad Abierta y Educación a Distancia, CUAED, Universidad Nacional Autonoma de Mexico. Mexico, D.F. Mexico. 